\title{
Virtualización y formación médica: reflexiones pedagógicas
}

\section{Resumen}

Este artículo, derivado del proyecto de investigación De las relaciones próximas a las interacciones virtuales: caso Facultad de Medicina, desarrollado en la Pontificia Universidad Javeriana de Bogotá, se propuso analizar las implicaciones pedagógicas de la incorporación de tecnologías de la información y la comunicación (TIC) en la formación médica, a partir de entrevistas con los distintos implicados en el escenario educativo de una facultad de medicina. La experiencia se interpretó en torno a categorías referidas a la relación pedagógica en la formación médica, la experiencia de aprendizaje y los tránsitos a la virtualidad.

Como resultado, fue ostensible la necesidad de considerar la virtualización cuando se piensa en la formación por medio de las TIC y en la necesidad de plantear un modelo pedagógico que sustente las nuevas relaciones educativas.

\section{Palabras clave descriptor}

Formación médica, aprendizaje en línea, método de enseñanza.

\section{Transferencia a la práctica}

Tradicionalmente, la formación del quehacer médico se ha fundamentado en una relación cara a cara, en la que el docente juega un papel protagónico en la transmisión de conocimientos, prácticas y valores. Con la incursión de las tecnologías y las mediaciones virtuales, han surgido nuevas formas de abordar la relación pedagógica en la formación médica. El presente artículo hace una reflexión sobre la necesidad de pensar una nueva matriz pedagógica para la formación médica que trascienda el uso instrumental de las tecnologías y la virtualidad, y genere otras relaciones con el conocimiento y entre los sujetos que participan en los procesos de virtualización, como nuevos escenarios de enseñanza y aprendizaje.

Para citar este artículo / To cite this article / Pour citer cet article / Para citar este artigo Solano-Murcia, M. (2015). Virtualización y formación médica: reflexiones pedagógicas. magis, Revista Internacional de Investigación en Educación, 7 (15), 49-62. 
Key words plus

Medical Training, Online Learning,

Teaching Method.

\section{Abstract}

This article, derived from the research project From close relationships to virtual interactions: Case study from the School of Medicine, developed at Pontificia Universidad Javeriana in Bogotá, discusses the pedagogical implications of incorporating information and communication technologies dash in medical training. This is based on interviews with the various stakeholders involved in the educational scenario of a medical school. The experience was interpreted around categories related to the pedagogical relationship in medical training, the learning experience and transits to virtuality. As a result, it was necessary to consider virtualization when thinking about education by using ICT, and consider the need to establish a pedagogical model that supports the new educational relationships.

\section{Transfer into practice}

Traditionally, medical training has been based on a face to face relationship, where the professor plays a key role in the transmission of knowledge, practices and values. With the incursion of technology and virtual mediations, there are new ways to address the pedagogical relationship in medical training. This paper reflects on the need to think a new teaching practice for medical training that goes beyond the instrumental use of technology and virtuality, and build other relationships between knowledge and subjects involved in the processes of virtualization, as new teaching and learning scenarios.
Mots clés descripteur

Formation en médecine, apprentissage en ligne, méthode d'enseignement.

\section{Résumé}

Cet article dérivé du projet de recherche Des rapports proches aux interactions virtuelles: un cas de la Faculté de Médecine, développé par la Pontificia Universidad Javeriana à Bogota, on propose faire une analyse des implications pédagogiques de l'incorporation de technologies de l'information et la communication dash dans la formation de médecins, à partir d'entretiens avec les différents impliqués dans le scenario éducatif d'une faculté de médecine. L'expérience a été interprétée par rapport aux catégories du rapport pédagogique dans la formation de médecins, l'expérience d'apprentissage et les transits à la virtualité. En tant que résultat, il a été ostensible le besoin de considérer la virtualisation lorsqu'on pense à la formation au moyen des TIC et au besoin d'exposer un modèle pédagogique qui soit le fondement des nouveaux rapports éducatifs.

\section{Transfert à la pratique}

Traditionnellement, la formation du travail du médecin a été fondée dans un rapport face à face, dans ce rapport l'enseignant joue un rôle protagoniste dans la transmission de la connaissance, les pratiques et les valeurs. Avec l'incursion des technologies et des médiations virtuelles, des nouvelles formes d'aborder le rapport pédagogique dans la formation des médecins ont surgisses. Le présent article fait une réflexion sur le besoin de penser une nouvelle matrice pédagogique pour la formation de médecins qui puise aller plus loin de l'usage instrumental des technologies et de la virtualité, en créant d'autres rapports avec la connaissance et entre les sujets participants dans les processus de virtualisation en tant que nouveaux scenarios d'enseignement et d'apprentissage.

\section{Palavras-chave descritor}

Formação médica, aprendizagem on-line, método de ensino.

\section{Resumo}

Este artigo derivado do projeto de pesquisa Das relações próximas às interações virtuais: caso Faculdade de Medicina, desenvolvido na Pontifícia Universidade Javeriana de Bogotá, propôs-se analisar as implicações pedagógicas da incorporação de tecnologias da informação e comunicação na formação médica, a partir de entrevistas com os diferentes envolvidos no cenário educativo de uma faculdade de medicina. A experiência foi interpretada em torno a categorias referidas à relação pedagógica na formação médica, a experiência de aprendizagem e os trânsitos à virtualidade.

Como resultado, foi ostensível a necessidade de considerar a virtualização quando pensar a formação através das TIC e a necessidade de colocar um modelo pedagógico para sustentar as novas relações educativas.

\section{Transferência para a prática} Tradicionalmente a formação do quefazer médico tem se fundamentado numa relação face a face, na que o docente joga papel protagonista na transmissão de conhecimento, práticas e valores. Com a incursão das tecnologias e mediações virtuais, surgiram novas formas de abordagem da relação pedagógica na formação médica.

O presente artigo faz reflexão sobre a necessidade de pensar uma nova matriz pedagógica para a formação médica que transcenda o uso instrumental das tecnologias e a virtualidade, e gere outros relacionamentos com o conhecimento e entre os sujeitos que participam dos processos de virtualização como novos cenários de ensino-aprendizagem. 


\section{Introducción}

El presente artículo esboza algunas reflexiones sobre los resultados de la investigación De las relaciones próximas a las interacciones virtuales: caso Facultad de Medicina, con el propósito de rescatar el hacer pedagógico en la formación médica poniendo en la mira los posibles desplazamientos que ocurren cuando se transita de las prácticas de formación tradicionales a las prácticas mediadas virtualmente.

Las categorías objeto de análisis en este artículo son la relación pedagógica en la formación médica, su dilema entre la permanencia y el cambio, la experiencia de aprendizaje: entre el saber y el saber-hacer y los tránsitos a la virtualización. Con estas categorías, se buscó comprender las maneras en que se establecen las interacciones docente-estudiante, las singularidades del proceso de enseñanza aprendizaje en este campo de formación y las exigencias pedagógicas para incursionar en la experiencia educativa mediada virtualmente.

En la investigación que dio origen a estas reflexiones se utilizó una metodología cualitativa que, de acuerdo con Julio Mejía-Navarrete (2002), "(...) permite descifrar la acción mediante el discurso, puesto que las acciones son siempre reflexivas. Es decir, incluyen el mundo de las representaciones simbólicas de los sujetos con que dan sentido a sus acciones" (p. 24).

Siguiendo esta perspectiva se utilizó la técnica de la entrevista individual en profundidad a 15 directivos y profesores de la Facultad de Medicina de la Pontificia Universidad Javeriana y del Hospital Universitario San Ignacio para hacer surgir, desde las subjetividades, las maneras en que los implicados se aproximan a las TIC en las tareas de docencia, dirección e investigación.

También se aplicaron técnicas de grupos focales y entrevistas colectivas a 250 estudiantes de pregrado y posgrado y administrativos para indagar el significado que otorgan a las nuevas tecnologías en los procesos de formación. En el análisis e interpretación se recurrió a un proceso de triangulación de fuentes de datos e investigadores.

La investigación proporcionó indicios sobre cómo la comunidad académica de esta facultad ha venido haciendo tránsitos de una práctica educativa sustentada en el cara a cara hacia una relación mediada virtualmente, para visibilizar las resistencias y movilidades de los procesos educativos. Este aspecto es significativo para los que están involucrados en este campo de conocimiento y práctica; más aún, cuando hay un consenso cimentado en la presencialidad como el espacio preferencial y, a su vez, el reconocimiento de la complementariedad de otras opciones educativas mediadas para dar calidad en los procesos de enseñanza-aprendizaje.

\section{La relación pedagógica en la formación médica: entre la permanencia y el cambio}

La medicina, del lat. medicina, es la ciencia y el arte de precaver y curar las enfermedades del cuerpo humano, según la Real Academia Española (2001). Como ciencia, construye un cuerpo de conocimiento que define sus paradigmas epistemológicos y métodos de abordaje. Como arte, es virtud, disposición y habilidad para hacer algo. No es un campo que se defina puramente científico, objetivo y positivo; es también una práctica enriquecida en y por las interacciones sociales y culturales y por los procesos subjetivos y humanos.

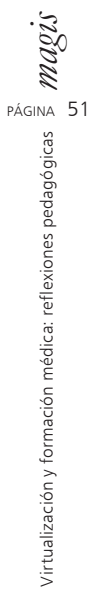

Descripción del artículo | Article description | Description de l'article | Artigo descrição

Este artículo de reflexión se deriva de la investigación De las relaciones próximas a las interacciones virtuales: caso Facultad de Medicina, que se desarrolló entre 2008 y 2010, en la Pontificia Universidad Javeriana, de Bogotá, financiada en el marco de la convocatoria Apoyo a proyectos de investigación en educación virtual, Pontificia Universidad Javeriana, Banco Santander, 2007. La autora se detiene en la relación pedagógica en la formación médica: entre la permanencia y el cambio; entre el saber y el saber-hacer, una experiencia de aprendizaje, ¿posible en lo virtual?, y los tránsitos a la virtualización. 
La Facultad de Medicina de la Pontificia Universidad Javeriana ha ido construyendo este campo de conocimiento mediante la definición de sus currículos, planes de estudios, prácticas educativas, investigaciones que promueve y las formas como concreta el perfil de formación profesional. En sus desarrollos, privilegia unos aspectos que, ante otras propuestas, definen la singularidad e identidad del profesional médico javeriano.

Desde sus inicios, la Facultad de Medicina dio cuenta de la preocupación por las formas de enseñar la medicina, la concepción de sujeto que aprende, el valor de la experiencia, los medios y recursos que conforman sus marcos de actuación. En últimas, la búsqueda de procesos pedagógicos que conducen al logro de sus propósitos educativos, interrelacionados cada vez más con los avances de la ciencia y las tecnologías médicas, las tecnologías de la comunicación e información, en el horizonte de los nuevos requerimientos y necesidades que la cultura va imponiendo, constituyen el horizonte actual de su acción pedagógica.

Así, por ejemplo, las memorias de los 50 años de la Facultad de Medicina recogen de manera amplia la historia y desarrollo de cada uno de los departamentos que la configuran hoy.

Uno de los testimonios dice:

(...) El curso de cirugía se inició en forma teórica, por los primeros meses de ese año, sin enfermos y salas de cirugía, organizando conferencias, seminarios, mesas redondas que suplieran la falta de pacientes. Esto produjo protesta por parte de los estudiantes, al cabo de los dos primeros meses, fecha en la cual, es decir en mayo de 1965, se inauguró el servicio y las salas de cirugía (Escallón, 1992, p. 70).

Llama la atención del relato que, si bien responde a un momento de inicio, se pueden evidenciar algunas características del modelo de formación de ese entonces: la presencia del paciente se configuraba desde ya fundamental en la formación médica, la interacción médico-pacienteestudiante daba sustento a la relación pedagógica para que se produjeran aprendizajes válidos; también aparece - aunque en menor valía - la importancia que se les otorgaba a los otros recursos educativos (conferencias, seminarios, etc.).

Hoy probablemente esa exigencia de interacción con pacientes reales, en los primeros años de formación, podría ser sustituida por pacientes simulados u otros recursos sin causar mayor zozobra en la comunidad académica.

La reflexión sobre la relación pedagógica en la formación médica en la Facultad de Medicina se ha expresado en una investigación cualitativa previa, cuyo propósito fue problematizar el aprendizaje en medicina, a propósito de la preocupación surgida en el equipo docente, ante la baja motivación y el escaso interés de los estudiantes en las actividades académicas, que se manifiestan en la inasistencia a las clases y en su poca participación durante ellas.

La búsqueda de respuestas a esa problemática permitió la comprensión de los aspectos de la formación básica y clínica y la tajante separación entre estas. También identificó los ritos de paso que marcan la iniciación e identificación con el mundo médico, como el uso de la bata, del fonendoscopio y los aspectos simbólicos de autoridad y reconocimiento. Además, se encontró que el ingreso a la clínica y sobre todo el contacto con el paciente son los que confieren el verdadero sentido a la formación médica y convierten el hospital en el lugar privilegiado de actuación (Santacruz, González, Beltrán \& Gómez, 1997). 
En general, el estudio mencionado reiteró la complejidad de la formación médica y planteó la necesidad de ir más allá de privilegiar la interacción docente-alumno en la apropiación del conocimiento, lo que muestra otras particularidades que dan significado al aprendizaje de la medicina y aporta categorías nuevas de análisis sobre este campo, como la relación pedagógica, los ritos de paso, el contacto con el paciente, el entorno y el imaginario.

Estos hallazgos, unidos a nuevas reflexiones sobre el quehacer docente en la Facultad de Medicina, en las nuevas condiciones y ambientes de enseñanza y aprendizaje permitieron seguir indagando sobre cuestiones como ¿cuáles son las particularidades que caracterizan la formación médica?, ¿qué constituye el núcleo de su ser y de su quehacer?, ¿qué aspectos son esenciales en la relación pedagógica?, ¿cuáles prácticas de la tradición permanecen? y ¿cuáles desplazamientos son posibles?

La respuesta a los anteriores interrogantes empieza por considerar la existencia de una tradición, en la que se han configurado unos códigos que determinan unas maneras de ser, estar y hacer la medicina; en otras palabras, de construir un sentido sobre esa práctica, manifiesto principalmente en la relación docente-estudiante-paciente en los procesos formativos.

En esa tradición, el docente es una figura "modelo" que enseña conocimiento y transmite valores que trascienden el mero ámbito de las actuaciones profesionales, éticas y morales. Tiene autoridad, poder y vocación, otorgado por la experiencia; su presencia en un mismo espacio y tiempo en la labor de enseñar, junto al estudiante que está aprendiendo, siempre se consideró indispensable, lo cual comienza a revaluarse en las nuevas condiciones educativas que aporta la tecnología. En ese sentido, la presencia del docente, si bien en algunos casos sigue siendo indispensable, en otros ya no es insustituible, como lo anticipa Julio Cabero (1996): “...Las Nuevas Tecnologías tienden a romper el aula como conjunto arquitectónico y cultural estable. El alumno puede interaccionar con otros compañeros y profesores que no tienen por qué estar situados en sus mismos contextos arquitectónicos" (p. 12).

Por su parte, el estudiante ha sido visto como un aprendiz, a quien le falta formarse, madurar, responsabilizarse y motivarse por la vocación médica; considerado en las conclusiones del estudio de Cecilia Escudero de Santacruz, Juan Carlos González, Nohora Beltrán y Gabriel Gómez (1997), el estudiante aún es percibido como un "adolescente", al que hay que guiar en su formación profesional, ética y moral. De otra parte, si se consideran como grupo, los estudiantes son generadores de tensiones originadas en el colectivo social, al constituir una fuerza instituyente de otras formas de entender la realidad y las experiencias de formación. Se puede percibir entonces que los estudiantes se ven a sí mismos como usuarios permanentes de las tecnologías. Así lo afirma un entrevistado: "Actualmente, a la presencialidad en la formación médica se le han ido incorporando otros elementos provenientes de las TIC; es así como se accede con relativa facilidad y frecuencia a fuentes de información, bases de datos, redes de conocimiento, publicaciones científicas, etc.". Además, su participación en redes sociales y el uso de los novedosos dispositivos electrónicos constituyen recursos para la comunicación y, subsidiariamente, para el aprendizaje.

En cuanto a los pacientes, esa tradición permite continuar viéndolos como los sujetos hacia los que se focalizan la intervención y los aprendizajes; además, al interactuar con ellos, se logra transferir, validar y confrontar lo aprendido en la formación médico científica; en síntesis, los pacientes constituyen la razón de ser de la formación médica. 
De esta manera, se puede ver que la tradición ha instituido unas formas de relacionarse entre los sujetos para construir los aprendizajes; es decir, de entablar una relación pedagógica' que puede tener distintos énfasis; por ejemplo, fundamentarse en el docente como sujeto de saber y figura central en el proceso; orientar el énfasis hacia el estudiante como el sujeto de aprendizaje teniendo en cuenta sus condiciones cognitivas o poner como centro de interés los escenarios y recursos que hacen posible los aprendizajes.

Esas tres tendencias se mueven en un contexto en el que actúan unos códigos reguladores de los vínculos interpersonales, establecidos sobre las asimetrías que se originan en las relaciones de poder; es decir, entre el docente, que detenta el conocimiento y la experiencia, y el aprendiz, sujeto en proceso de entrenamiento. En ese contexto de relaciones asimétricas, no hay que olvidar que, aunque haya una sujeción del estudiante, también es posible para este configurar y recrear sus propias maneras de habitar esos espacios.

Junto a la mirada tradicional de la formación médica, se han ido construyendo nuevos recursos que reclaman otra mirada. Los avances científicos y tecnológicos y las nuevas demandas socioculturales jalonan transformaciones que movilizan las formas tradicionales de interactuar. Las tecnologías médicas para garantizar la confiabilidad de sus diagnósticos, los tratamientos e intervenciones, las tecnologías de la comunicación y la información y la conectividad crean posibilidades para un mundo global y una nueva sociedad del conocimiento, lo que pone a cada uno de los sujetos de la relación en nuevos lugares frente al conocimiento. Aunque los estudiantes entrevistados consideran que: "en contenido virtual, como programas, la facultad está apenas entrando en el mundo de las inscripciones a los estudiantes a estos programas, pero es una puerta a un mundo de interactividad y de simulación muy útil para todos nosotros", y que, por otra parte, la tecnología "permite obtener mayor información y realizar algunos procesos de simulación", lo cual ha parecido adecuado.

Parece encontrarse en estos testimonios de estudiantes y de algunos docentes la no distinción entre conocimiento e información. Antonio Sánchez-Asín, Josep Lluís Boix-Peinado y Pedro Jurado de los Santos (2009) enfocan esta distinción en los siguientes términos:

(...) Insistir en la diferencia entre "información" como acumulación o almacenamiento de datos y "conocimiento"; es decir, la inteligente comprensión, interpretación y metacognición de lo adquirido, a través de una adecuada selección y procesamiento de dicha información. El conocimiento trata de la interiorización de la información susceptible de ser utilizada, de proveer la acción. Se refiere a los hechos o experiencia conocida por las personas, conscientemente adquirida a través de la experiencia (p. 184).

En la actualidad, el conocimiento y la información circulan de múltiples maneras, no hay lugares fijos. La comunicación docente, de acuerdo con Alicia Susana Francia (2005), empieza a recibir los efectos del anclaje de lo virtual: "la comunidad actual no es comunidad de sentido, sino comunidad virtual" (p. 2); así, los jóvenes reconfiguran el lugar de los saberes

1 La pedagogía, como reflexión personal y dialogal sobre las acciones educativas, se refiere a las actividades del profesor en relación con los alumnos y sus respectivos microentornos y al aprendizaje que hace posible al estudiante conformar una mentalidad y actitudes creativas. Tomada de Pontificia Universidad Javeriana, PUJ (1992). Proyecto Educativo, artículo 19. 
y sus relaciones con quienes durante mucho tiempo detentaron el dominio del saber, como los docentes.

En esta perspectiva, los procesos de formación médica están en una paradoja entre el permanecer y el cambio, entre el conocimiento y la información, entre la tradición y la actualidad, entre las reglas únicas y la diversidad de posibilidades, entre la relación directa y la relación virtual. La superación de las tensiones que estas dualidades generan se ubica en el corazón de la reflexión sobre el devenir de la formación médica, que parece avizorar que lo uno puede coexistir con lo otro, como medio para recrear y enriquecer lo ya existente aportado por la experiencia dentro de un proceso, que, antes de causar antagonismos, lleve a procesos de complementariedad.

\section{Entre el saber y el saber-hacer, ¿una experiencia de aprendizaje posible en lo virtual?}

Como se mencionó antes, un principio compartido colectivamente por la comunidad académica en cuestión es que el aprendizaje de la medicina necesariamente tiene que ser presencial, cara a cara. Es decir, una experiencia que involucre todos los sentidos: ver, palpar, tocar, oler, para tener mayor incidencia con el paciente, un estar consciente en relación con un otro en un mismo espacio y tiempo. Como experiencia de aprendizaje, el cara a cara es la posibilidad de construir un saber-hacer, guiado y garante de aprendizajes seguros y confiables.

La posibilidad de leer en la interacción con el paciente los signos, indicios y sospechas que participan en la semiosis médica es parte de ese saber-hacer, surgido de la relación entre la teoría y práctica.

En las entrevistas realizadas, unánimemente, los docentes privilegiaron la relación cara a cara como fundamental en las relaciones entre docentes y estudiantes que están en proceso de formación médica. Sobre el particular, se señalan algunas de las ideas expresadas por los docentes y directivos en las entrevistas:

La función de la formación médica necesariamente tiene que ser presencial, uno no aprende la relación médico-paciente mientras no la ve y la realiza.

La relación que se establece en el entrenamiento de nuevos profesionales, entre el paciente, el estudiante y el profesor, interacción de tres, es vital para la adquisición de habilidades.

La presencialidad es importante en el desarrollo de actitudes, más que para los conocimientos mismos; eso no se va a poder sustituir con medios virtuales.
Vemos en estas afirmaciones que la interacción directa entre médico, paciente y estudiante se considera insustituible, ya que en el trato presencial se crea el clima de confianza en la comunicación y el nivel de seguridad para la atención y resolución de las dificultades en la formación médica.

La relación que se establece con estos recursos está basada en un uso funcional e instrumental. En otros términos, se trata de un aprendizaje con la tecnología que tiene efectos en el pensamiento, en el campo de las representaciones, y además:

(...) afectan específicamente lo que se ha descrito como marcos de pensamiento. Estos vendrían a ser un conjunto de representaciones mentales - no estructuras constantes- que implican estrategias de pensamiento, estrategias de metacognición, formas de ver el mundo y ciertas habilidades de procesamiento y comunicación de la información, que efectivamente sirven de guía, apoyando y organizando el proceso de aprendizaje (Suárez, 2003, p. 7, que cita a Gavriel Salomon, 1992).

El uso que en primera instancia puede manifestarse como una habilidad tecnológica de los estudiantes es reconocido por los profesores como una competencia frente a lo tecnológico en la que ellos se sienten en desventaja. Según Alejandro Piscitelli (2009), la relación entre el nativo/inmigrante digital ha establecido una distancia tecnológica intergeneracional y un cambio en las relaciones de poder frente a las TIC, como se traduce en lo expresado por los entrevistados:

Se siente temor por parte de los docentes por el analfabetismo tecnológico, por los retos de la virtualidad y la pérdida del protagonismo.

Hay cierta resistencia de parte de los profesores, hay una mentalidad primitiva de que la información se posee, uno la posee.

El paradigma cambia porque el profesor antes era la fuente de conocimiento, él estaba como el nicho de conocimiento que iba a guiar.

Ahora de pronto el estudiante llega con mejores datos o más actualizados que el profesor.

Las formas de interacción con la tecnología y sus repercusiones en los procesos de enseñanza aprendizaje conllevan la ampliación y transformación de los escenarios en que se produce la relación docente: las aulas de clase y el hospital, lugares tradicionales de la formación médica. La posibilidad de aprender no se limita a esos espacios, sino que la amplia circulación 
y la posibilidad de construir saberes desbordan los campos simbólicos y materiales de los escenarios de aprendizaje y trascienden la labor docente, al problematizarla y enfocar la discusión en los procesos de aprendizaje en general para particularizar lo que sucede con la introducción de las herramientas virtuales. Los estudiantes consideran necesario que tanto docentes como estudiantes incursionen cada vez más en el uso de las herramientas tecnológicas para mejorar su formación: "Los docentes deben entrenarse, conocer y manejar los diferentes escenarios de aprendizaje, entre los cuales está la virtualidad, y nosotros, los estudiantes, hacer uso de la virtualidad como herramienta de conocimiento".

Desde el punto de vista del aprendizaje, un primer aspecto por revisar es la noción de mediación. Mediar es un concepto que evoca estar en medio de algo o intercediendo por algo o alguien, del lat. mediāre. Es un concepto polisémico y su aplicación difiere en los distintos campos. Hay una fuerte tendencia a asociar y reducir la mediación al uso de artefactos. Según Beatriz Fainholc (2004), la mediación tecnológico-educativa "no remite a los artefactos sino a los nuevos modos de percepción y lenguaje, nuevas narrativas, escrituras y sensibilidades que configuran las subjetividades" (p. 2).

Es decir, los procesos de aprendizaje presencial o virtual se sustentan en acciones mediadas, no restringidas al uso de medios comunicativos, como las plataformas virtuales, sino a un nuevo campo de relacionamiento con el conocimiento, con la cultura, con la creación de lenguajes, con los procesos de interacción entre el estudiante y el docente. Esta manera de entender la mediación permite crear nuevas formas de comunicación, de diálogo, de simbolización y de representación de la realidad.

Desde esta perspectiva, conocemos el mundo mediadamente. $Y$ para ello nos valemos de distintos recursos físicos y culturales. Nos acercamos a una realidad de manera interpretada. En los procesos educativos, así se esté frente a frente con un docente, él está ejerciendo una mediación, proporcionando a sus estudiantes una interpretación para favorecer el aprendizaje (Parra, 2007).

En el campo educativo, se ha trabajado ese asunto. En la perspectiva de Daniel Prieto-Castillo (2004), se enfatiza la dimensión pedagógica con el tratamiento de los contenidos, las prácticas de aprendizaje, la forma de los materiales, las relaciones presenciales, las búsquedas en el texto y en el contexto, entre otras posibilidades. O, como reconoce Ángel H. Facundo (2004): "toda educación es mediada" (p. 5).

Al resignificar el concepto de mediación, la pedagogía y la didáctica recobran su valor. Esa resignificación se hizo más patente desde el momento en que se configuraron las experiencias educativas a distancia, la creación de ambientes de aprendizaje y la elaboración de materiales didácticos con la ayuda de la tecnología. Todo ello ha contribuido a promover los aprendizajes con la acción mediada de nuevos elementos que aparecen en el escenario educativo.

Otro elemento para considerar en la comprensión de la incorporación de tecnologías para la mediación de los procesos educativos es la perspectiva de Lev Vygotsky, quien hace hincapié en una doble estructuración de los procesos cognitivos del aprendizaje, que se influyen mutuamente. Esa doble articulación se caracteriza por procesos externos e internos; los primeros se inician como una actividad interpersonal con el mundo exterior de las relaciones, los contextos, la cultura y los dispositivos pedagógicos.

Para que esta experiencia se convierta en aprendizaje debe constituirse luego en una experiencia intrapersonal, es decir, debe suceder un proceso de interiorización, de representación interna. "Ello significa simplemente que algunas categorías de funciones mentales superiores (atención voluntaria, memoria lógica, pensamiento verbal y conceptual, emociones complejas, etc.) no podrían surgir y constituirse en el proceso del desarrollo sin la contribución constructora de las interacciones sociales" (Ivic, 1994, p. 4).

En el caso de la educación mediada con la ayuda de las tecnologías, la relación pedagógica se estructura externa e internamente, según la perspectiva vygotskyana:

(...) de un modo definido otorgando a los sujetos formas de actuación externa para el aprendizaje, pero a su vez, a partir de esa misma estructura y atributos tecnológicos, promueve en el sujeto una modificación interna de sus estrategias de pensamiento y aprendizaje. Esta doble orientación, externa e interna, atribuible a los instrumentos de mediación, debe representar otro punto de inflexión en el análisis y lectura pedagógica de las nuevas tecnologías en la relación educativa (Suárez, 2003, p. 1).

En este sentido, cuando la labor docente se fundamenta en la acción mediadora para el aprendizaje, haciendo uso de la pedagogía y los recursos didácticos, se está ejerciendo una mediación con el fin de proveer y favorecer el aprendizaje. De alguna manera, los docentes avizoran la necesidad de hacer una intervención pedagógica cuando afirman que ellos mismos "deberían filtrar ese tipo de recursos y guiar a los estudiantes hacia el análisis ético".

Para el caso de la interacción médico-estudiante, esta manera de asumir la relación pedagógica hace que la presencia corporal — tanto de los aprendices como de los maestros - trascienda las intenciones 
informativas y la adquisición de conocimientos, para dar paso a experiencias de aprendizaje no verbales, valorativas, actitudinales, éticas, legales y de relacionamiento con el paciente. Como se señala:

(...) La efectividad comunicativa y pedagógica no depende necesariamente de la condición de presencialidad que caracteriza la interacción social cara a cara, sino de la organización de un conjunto de experiencias educativas que ponen a disposición de un estudiante los mejores recursos culturales para el aprendizaje (Parra, 2007, p. 12).

Por ello, es necesario repensar las formas de aprender en la formación médica como un asunto complejo, que no se reduce a lograr unos resultados de aprendizaje, sino a resaltar que se aprende dentro de un proceso, en el que se van desarrollando unas competencias manifestadas en saberes, acciones, sentires y valores con los que se construye el sujetomédico en formación.

La incorporación de otros recursos didácticos puede contribuir a que la formación médica adopte una pedagogía en la que aprender significa más que repetir un conocimiento, emular una práctica y aplicar un saber. Tiene que ver con comprender, recrear, resolver, resignificar en las diferentes situaciones a las que se ve enfrentado, recurriendo a lo que se ha adquirido y lo nuevo por indagar. Como lo manifiestan los miembros de esa comunidad educativa:

Todos deben contribuir no solo para aprovechar lo que se encuentra en la Web, sino para mejorar la calidad de esta. Enseñar lo necesario para poder utilizarlo y nunca olvidar que es solo una herramienta más, sin dejar a un lado la relación médico-paciente.

En últimas, es necesario que el estudiante logre sobrepasar las fronteras de lo dado, aprender a aprender como un recurso potenciado de su experiencia ínter e intrapersonal.

Sin embargo, es importante pensar la relación cara a cara no como algo que por sí mismo es suficiente, sino que - al ser parte central de la pedagogía - requiere estar revestido de un proceso de organización didáctica que conduzca a los aprendizajes por el saber, saber-hacer, saber comunicar y saber interactuar, entre otros; sin ser excluyente de otras formas de aprender. Pensar en la búsqueda de nuevos conceptos movilizadores que, como señala Ángel H. Facundo (2004), conduzcan a romper modelos o paradigmas pedagógicos tradicionales y a descubrir que el "aprendizaje ya no puede seguir siendo una 'consecuencia' de la enseñanza, sino un proceso fundamentalmente autónomo, de autodescubrimiento, realización y transformación" (p. 7).

En otras palabras, en la formación médica, el proceso de aprendizaje es polimorfo en recursos, para que este ocurra. Así, por ejemplo, es colaborativo, puesto que se aprende de y con los docentes, de y con los pacientes y de los compañeros; se produce cara a cara pero también en una relación mediada tecnológicamente por el uso de simuladores, biotecnologías, bases de datos, libros impresos y digitales, artículos científicos, consultas e interconsultas online, historias de vida electrónicas, teleconferencias especializadas y la participación en experiencias de telemedicina y programas de e-Health, etc., que no solamente proporcionan información, sino que posibilitan la interacción cognitiva planteada para lograr aprendizajes efectivos. Los elementos mencionados son cada vez más frecuentes en la formación, lo cual refuerza la necesidad de asumir esos usos para 
crear nuevas formas de aprendizaje que modifiquen las tradicionales maneras de abordar la formación. Consiste en:

Aprovechar la potencialidad de estas tecnologías para impulsar nuevas formas de aprender y enseñar. No se trata ya de utilizar las TIC para hacer lo mismo pero mejor, con mayor rapidez y comodidad o incluso con mayor eficacia, sino para hacer cosas diferentes, para poner en marcha procesos de aprendizaje y de enseñanza que no serían posibles en ausencia de las TIC (Coll, 2009, p. 124).

No obstante, ¿qué tan cercanas son las posibilidades de integrar en las prácticas de la formación médica las mediaciones pedagógicas, tecnológicas y comunicativas en la Facultad de Medicina?

\section{Los tránsitos a la virtualización}

Los docentes entrevistados se aproximan a la virtualidad. Algunos la aprecian como "un recurso de gran utilidad y apoyo en la enseñanza y formación de los estudiantes, principalmente en la accesibilidad al conocimiento, información rápida, conectividad y medio para la sustitución de algunas actividades docentes". Para otros, "lo virtual (...) parece buenísimo para revisar un caso, para discutir un tema, para unirse con otras instituciones y hacer una teleconferencia sobre un caso clínico, para ver una cirugía donde usted pone allá la teleconferencia o en el medio virtual y la gente está viendo, pero el estudiante no va a aprender a operar en el medio virtual". "Los simuladores (...) facilitan el desarrollo de muchas destrezas, de muchas competencias, digamos que sin incurrir en el riesgo o en la acción insegura con el paciente". O como lo plantea Pío Iván Gómez (2003), en el entrenamiento médico se empieza a valorar el uso de simuladores en las intervenciones directas médico-quirúrgicas de estudiantes principiantes, por las implicaciones médico legales en los imprevistos que pueden surgir.

Las anteriores son afirmaciones que recuerdan las tipologías descritas en el estudio de Helena Segura-Badia (2006) sobre experiencias de actualización médica a distancia y las maneras como la comunidad académica se aproxima a esta modalidad: a) como una forma de provisión de contenidos (enfoque tradicional), b) como gestión de contenidos (reconoce que la educación es más que una distribución de contenidos) y c) online, que da cabida a la interacción y comunicación para lograr desarrollar aprendizajes.

Por otra parte, la autora visibiliza la incertidumbre que causa pensar la incorporación de acciones educativas virtualizadas en el devenir de la formación médica. En ese sentido, los testimonios de los estudiantes muestran que la incorporación de la tecnología "ha ido cambiando progresivamente, de modo que cada vez aumenta el contacto con computadores y sistemas electrónicos y disminuye el contacto con la información en papel". "Todos estamos involucrados en este cambio. Los docentes también tienen acceso a la información desde cualquier lugar. Los pacientes pueden conocer más acerca de su enfermedad y el proceso terapéutico". No obstante, consideran que "la virtualidad debe ponerse en su lugar y los docentes principalmente deben saber usarla para facilitar el aprendizaje de sus estudiantes"; sin embargo, "la virtualidad no debe sustituir la docencia, pues esta es primordial, es la que mayor enseñanza deja a la hora de traducir en un lenguaje claro y concreto algo que muchos textos, videos y artículos no logran". 
Efectivamente, la incorporación de las TIC en procesos de formación trae consigo nuevas nociones espacio-temporales que modifican lo establecido, lo instaurado y lo adoptado por un grupo humano en el contexto médico clínico e introducen nuevas formas de aprender, comunicarse y configurar la realidad; pero también trae dilemas entre la aceptación y las reservas, entre la utilidad y las amenazas, entre los riesgos y sus posibilidades. Tanto las nuevas nociones como los dilemas surgidos plantean la necesidad de disponer de un abordaje pedagógico-didáctico característico de los procesos de virtualización de la educación. Como observa Jaime Parra-Rodríguez (2007), se trata de "constituir mundos virtuales que se caracterizan por las posibilidades de representación y creación de nuevas realidades que no se configuran por objetos tangibles, sino por objetos simbólicos" (p. 23).

Pensar la virtualización de los procesos educativos en la formación médica - más que cifrarla en la utilización de tecnologías digitales, o en creer que estas realicen los procesos educativos - significa problematizar las formas instituidas de educar y una revisión de los anteriores paradigmas. Implica una "inversión" conceptual en materia de pedagogía y de educación (Facundo, 2004). La virtualización, como lo anotan Miquel Domènech y Francisco Javier Tirado (2002), no es más que el movimiento de "convertirse en otro"; es algo dinámico, y ese movimiento implica irreversibilidad, ya que transforma la actualidad inicial en un caso particular de una problemática más general o en un caso particular de un plano diferente que queda englobado y redefinido en el campo problemático que abre la virtualización.

Desde nuestra experiencia de docencia virtual, consideramos la virtualización en el campo pedagógico como una acción mediadora hecha por el docente para pensar y crear distintos escenarios de formación con el fin de potenciar los aprendizajes; como la posibilidad de creación de realidades educativas, de nuevos mundos de aprendizajes y de relaciones entre los actores del proceso educativo mediante acciones deliberadas, dinámicas, planeadas y organizadas dentro de un marco o programa; en este caso, el de la formación médica. Esto correspondería en términos generales a lo planteado por Pierre Lévy (1999), para quien "virtualizar una entidad cualquiera consiste en descubrir la cuestión general a la que se refiere, en mutar la entidad en dirección a este interrogante y en redefinir la actualidad de partida como respuesta a una cuestión particular" (p. 19).

Si, como lo dice este mismo autor (p. 20), "la virtualización es uno de los principales vectores de la creación de realidad", en la experiencia formativa de la facultad esa nueva realidad se ha empezado a construir mediante la incorporación de tecnologías que derivan en usos y prácticas pedagógicas, tales como los simuladores, la creación de ambientes virtuales, el uso de plataformas virtuales, la producción de CD interactivos como iniciativas pedagógicas de sus docentes, la oferta de programas a distancia y virtuales, como es el caso de las especializaciones en Gestión Aplicada a Servicios en Salud y en Prevención del Maltrato Infantil, entre otras, así como en la vida cotidiana la actividad académica ya no se concibe sin computadores, Internet, correo electrónico, redes sociales virtuales y teléfonos celulares, entre otros.

Pero aún persisten los temores y la desconfianza en su efectividad frente a la relación de formación directa con el estudiante y el paciente. La presencialidad sigue ocupando un lugar preponderante. Sin embargo, se abren posibilidades para simbolizar otras formas de relación, como Pío Iván Gómez (2003) afirma, y aunque nada podrá reemplazar la interacción con el paciente real en el aprendizaje, los pacientes simulados pueden contribuir a preparar al estudiante para cuando se encuentre frente a la realidad de los pacientes a tratar. En este mismo sentido, el estudio de Ariel Iván Ruiz-Parra, Edith Ángel-Müller y Óscar Guevara (2009) concluye:

En el desarrollo de tecnologías como la simulación clínica, el aprendizaje virtual puede complementar la enseñanza, facilitar el aprendizaje y mejorar en los estudiantes habilidades clínicas, comunicativas, de trabajo en grupo y de respuesta ante situaciones de urgencia [...]; sin embargo, no sustituyen los escenarios clínicos reales ni el aprendizaje directo con los pacientes.

A lo anterior hay que agregar que en el aprendizaje virtual hay un potencial interactivo y comunicativo entre docentes y estudiantes en la consumación del acto de aprender. Albert Sangrà (2001) complementa estos criterios:

En la virtualidad deben cumplirse unos mínimos, ya que la gran diferencia entre lo presencial y la no presencialidad reside en que esta necesita de la explicación de los procesos y de las metodologías de aprendizaje, más que la presencialidad, y por ello la tarea debe estar siempre previamente diseñada y debe ser conocida por el estudiante (p. 119).

Además, su inmaterialidad, interactividad, autonomía y digitalización amplían las posibilidades de incorporación significativa de la virtualidad en la formación médica, sin que por ello se produzca la eliminación o sustitución de la tradición cara a cara (Jaime Parra-Rodríguez, 2007; Helena Segura-Badia, 2006; y Nilson A. Contreras-Carreto, 2006). 
Para avanzar en la virtualización se requiere repensar las relaciones generadas durante el proceso de la formación médica, entre otras, las de poder y las de reconocimiento del otro; plantear desafíos, interrogantes y nuevas formas de aprender; apropiarse de nuevas relaciones con el conocimiento y de circulación de la información; reflexionar sobre la incorporación de tecnologías, aprendiendo de ellas y con ellas (Suárez, 2003).

A ello hay que agregar, sin desconocer el valor de los saberes especializados en la formación médica, que la base de las transformaciones se construye desde el valor y el reconocimiento que se le dé a la docencia en sí misma. Muchas veces, como lo señala Miguel Ángel Zabalza (2009), esta docencia no constituye un asunto relevante en la tradición universitaria reciente, dándose prioridad a otros aspectos, como ser líder en investigación o tener influencia sociocultural y política.

\section{Conclusiones}

La investigación señaló que en la Facultad de Medicina de la Pontificia Universidad Javeriana, a pesar de su tradición predominantemente presencial en los procesos de formación, en la actualidad se plantea un tránsito progresivo en la incorporación de las TIC con propósitos educativos.

Desde el punto de vista pedagógico, se encontró que la aproximación al tema de las TIC genera controversia, pues marca posiciones de posibilidad y adopción de manera selectiva. En este sentido, dada la complejidad y variedad de competencias propias de la formación del saber-hacer médico, frente a las cuales se prefiere mantener la relación pedagógica normalizada, las TIC son aceptadas como una estrategia de apoyo educativo de información y comunicación.

De la misma manera, se hizo evidente la necesidad imperiosa de la reflexión sobre el modelo pedagógico que subyace en la mediación virtual durante la formación, con el propósito de suscitar nuevas formas de enseñar y aprender. De ese modo, se garantizará avanzar en el proceso de virtualización que permita trascender lo meramente instrumental y trabajar en la perspectiva de asegurar la calidad de los procesos pedagógicos mediados virtualmente para brindar confianza a los distintos miembros de la comunidad educativa.

\section{Sobre la autora}

Martha Solano-Murcia es profesora asociada. Departamento de Pediatría. Facultad de Medicina de la Pontificia Universidad Javeriana. Miembro del grupo de investigación Infancias, Relaciones y Transformaciones Sociales. Pontificia Universidad Javeriana, Bogotá, D.C.

\section{Referencias}

Cabero, J. (1996). Nuevas tecnologías, comunicación y educación. Edutec: Revista Electrónica de Tecnología Educativa, 1. Disponible en: http:// edutec.rediris.es/Revelec2/Revelec1/revelec1.html

Coll, C. (2009). Aprender y enseñar con las TIC: expectativas, realidades y potencialidades. En Roberto Carneiro, Juan Carlos Toscano \& Tamara Díaz (coords.). Los desafíos de las TIC para el cambio educativo, 113126. Disponible en: http://www.oei.es/metas2021/LASTIC2.pdf

Contreras, N. (2006). Bases tecnopedagógicas de la educación médica virtual. Análisis de un nuevo paradigma en la educación médica. Médica 
Sur, México, 13 (1, enero-marzo), 7-11. Disponible en: http://www. medigraphic.com/pdfs/medsur/ms-2006/ms061b.pdf

Domènech, M. \& Tirado, F. (2002). Lo virtual y lo social. Athenea Digital, 1, 1-9. Disponible: http://www.raco.cat/index.php/Athenea/article/ viewFile/34092/33931

Escallón, A. (1992). Historia del Departamento de Cirugía de la Facultad de Medicina de la Universidad Javeriana y del Hospital Universitario San Ignacio. Universitas Médica, Facultad de Medicina 50 años, 33, 2-3 (abril-septiembre), 69-74.

Escudero de Santacruz, Cecilia; González, Juan Carlos; Beltrán, Nohora \& Gómez, Gabriel (1997). La relación pedagógica en la formación médica. Aproximación a la perspectiva de los estudiantes. Informe inédito Facultad de Medicina de la Pontificia Universidad Javeriana, Bogotá.

Facundo, Á. H. (2004). La virtualización desde la perspectiva de la modernización de la educación superior: consideraciones pedagógicas. Revista Universidad y Sociedad del Conocimiento, 1 (1). Disponible en: http://www.uoc.edu/rusc/dt/esp/facundo1104.pdf

Fainholc, B. (2004). El concepto de mediación en la tecnología educativa apropiada y crítica. Disponible en Educar, el portal educativo del Estado argentino: http://portal.educ.ar/debates/educacionytic/ nuevos-alfabetismos/el-concepto-de-mediacion-en-la-tecnologiaeducativa-apropiada-y-critica.php

Francia, A. (2005). Desde la subjetividad pedagógica tradicional a la virtual. Revista Iberoamericana de Educación, 35 (5). Disponible en: http:// www.rieoei.org/deloslectores/874francia.PDF

Gómez, P. I. (2003). Uso de simuladores y otras ayudas educativas en medicina. Revista Facultad de Medicina de la Universidad Nacional de Colombia, 51 (4), 227-232.

Ivic, I. (1999). Lev Semionovich Vygotsky (1896-1934). Perspectivas: Revista Trimestral de Educación Comparada (París, UNESCO, Oficina Internacional de Educación), XXIV (3-4), 773-799. Disponible: http://www. ibe.unesco.org/publications/ThinkersPdf/vygotskys.PDF

Lévy, P. (1999). ¿Qué es lo virtual? Barcelona: Paidós.

Mejía, J. (2002). Perspectiva de la investigación social de segundo orden. Cinta de Moebio, Revista Electrónica de Epistemología de Ciencias, Facultad de Ciencias Sociales, Universidad de Chile, 14, 1-34. Disponible en: http://www.redalyc.org/articulo.oa?id=10101405

Parra, J. (2007). Aprendizaje y conectividad. Bogotá: Pontificia Universidad Javeriana.

Piscitelli, A. (2009). Nativos e inmigrantes digitales: una dialéctica intrincada pero indispensable. En Roberto Carneiro, Juan Carlos Toscano \& Tamara Díaz (coords.). Los desafíos de las TIC para el cambio educativo, 71-78. Disponible en: http://www.oei.es/metas2021/LASTIC2.pdf

Pontificia Universidad Javeriana, PUJ (1992). Proyecto Educativo. Disponible en: http://www.javeriana.edu.co/puj/documentos/proyecto.htm

Prieto, D. (2004). Sobre la necesidad de la mediación pedagógica de las tecnologías. Disponible en: http://www.monografias.com/trabajos913/ sobre-necesidad-mediacion/sobre-necesidad-mediacion.shtml

Real Academia Española (2001). Diccionario de la lengua española [versión electrónica]. 22a ed. Madrid: Real Academia Española. Disponible: www.rae.es

Ruiz-Parra, A.; Ángel-Müller, E. \& Guevara, Ó. (2009). La simulación clínica y el aprendizaje virtual. Tecnologías complementarias para la educación médica. Revista Facultad de Medicina de la Universidad Nacional de 
Colombia, 57 (1), 67-79. Disponibleen: http://www.scielo.org.co/scielo. php?script=sci_arttext\&pid=S0120-00112009000100009\&lng =

Salomon, G. (1992). Las diversas influencias de la tecnología en el desarroIlo de la mente. Infancia y Aprendizaje, 58, 143-159.

Sangrà, A. (2001). Enseñar y aprender en la virtualidad. Educar, Universitat Oberta de Catalunya, 28, 117-131. Disponible en: http://ddd.uab.es/ pub/educar/0211819Xn28p117.pdf

Sánchez, A.; Boix, J. \& Jurado, P. (2009). La sociedad del conocimiento y las TICs: Una inmejorable oportunidad para el cambio docente. Pixel-Bit. Revista de Medios y Educación, 34, 179-204. Disponible en: http:// www.sav.us.es/pixelbit/pixelbit/articulos/n34/13.pdf

Segura, H. (2006). Las nuevas tecnologías y la formación médica continuada a distancia. Educación Médica, 9 (3), 118-126. Disponible en: http://scielo.isciii.es/pdf/edu/v9n3/original4.pdf

Suárez, C. (2003). Los entornos virtuales de aprendizaje como instrumento de mediación. Ediciones Universidad de Salamanca. Disponible en: http://campus.usal.es/ teoriaeducacion/rev_numero_04/n4_art_ suarez.htm,webmrte@usal.es

Zabalza, M. Á. (2009). Ser profesor universitario hoy. La Cuestión Universitaria, 5, 69-81. Disponible en: http://tecnologiaedu.us.es/mec2011/ $\mathrm{htm} / \mathrm{mas} / 3 / 31 / 47 . p d f$ 